\title{
ORIGINAL
}

\section{Integrative omics provide biological and clinical insights into acute respiratory distress syndrome}

Mulong Du1,2, Joe G. N. Garcia ${ }^{3}$, Jason D. Christie ${ }^{4}$, Junyi Xin ${ }^{5}$, Guoshuai Cai ${ }^{6}$, Nuala J. Meyer ${ }^{4}$, Zhaozhong Zhu', Qianyu Yuan', Zhengdong Zhang ${ }^{5}$, Li Su', Sipeng Shen ${ }^{2,7}$, Xuesi Dong ${ }^{1,2,7}$, Hui Li ${ }^{8}$, John N. Hutchinson ${ }^{8}$, Paula Tejera', Xihong Lin ${ }^{8,9}$, Meilin Wang ${ }^{5,10^{*}}$, Feng Chen ${ }^{2,7^{*}}$ and David C. Christiani ${ }^{1,11^{*}}$ (D)

(c) 2021 Springer-Verlag GmbH Germany, part of Springer Nature

\begin{abstract}
Purpose: Acute respiratory distress syndrome (ARDS) is accompanied by a dysfunctional immune-inflammatory response following lung injury, including during coronavirus disease 2019 (COVID-19). Limited causal biomarkers exist for ARDS development. We sought to identify novel genetic susceptibility targets for ARDS to focus further investigation on their biological mechanism and therapeutic potential.

Methods: Meta-analyses of ARDS genome-wide association studies were performed with 1250 cases and 1583 controls in Europeans, and 387 cases and 387 controls in African Americans. The functionality of novel loci was determined in silico using multiple omics approaches. The causality of 114 factors potentially involved in ARDS development was assessed using Mendelian Randomization analysis.

Results: There was distinct genetic heterogeneity in ARDS between Europeans and African Americans. rs 7967111 at 12p13.2 was functionally associated with ARDS susceptibility in Europeans (odds ratio $=1.38 ; P=2.15 \times 10^{-8}$ ). Expression of two genes annotated at this locus, BORCS5 and DUSP16, was dynamic but ultimately decreased during ARDS development, as well as downregulated in immune cells alongside COVID-19 severity. Causal inference implied that comorbidity of inflammatory bowel disease and elevated levels of C-reactive protein and interleukin-10 causally increased ARDS risk, while vitamin D supplementation and vasodilator use ameliorated risk.
\end{abstract}

Conclusion: Our findings suggest a novel susceptibility locus in ARDS pathophysiology that implicates BORCS5 and DUSP16 as potentially acting in immune-inflammatory processes. This locus warrants further investigation to inform the development of therapeutic targets and clinical care strategies for ARDS, including those induced by COVID-19.

Keywords: ARDS, COVID-19 severity, Biomarkers, Causal inference, Multi-omics

\footnotetext{
*Correspondence: mwang@njmu.edu.cn; fengchen@njmu.edu.cn; dchris@hsph.harvard.edu

${ }^{1}$ Department of Environmental Health, Harvard T.H. Chan School

of Public Health, Harvard University, 655 Huntington Avenue, Boston, MA

02115, USA

2 Department of Biostatistics, Center for Global Health, School

of Public Health, Nanjing Medical University, 101 Longmian Avenue,

Nanjing 211166, Jiangsu, China

${ }^{5}$ Department of Genetic Toxicology, The Key Laboratory of Modern Toxicology of Ministry of Education, Center for Global Health, School of Public Health, Nanjing Medical University, 101 Longmian Avenue, Nanjing 211166, Jiangsu, China

Full author information is available at the end of the article
}

\section{实 Springer}




\section{Introduction}

Acute respiratory distress syndrome (ARDS) is a type of hypoxemic respiratory failure in patients with conditions that predispose to or cause lung injury [1]. ARDS produces a high mortality rate $(30-40 \%)$ in hospitals worldwide [2]. Incidence of this condition is increasing sharply with the ongoing coronavirus disease 2019 (COVID-19) pandemic [3], with a median onset time of 8-12 days after COVID-19 symptom onset $[4,5]$.

Multi-omics approaches identify biomarkers for disease etiology and therapeutics [6]. Previously, we summarized 201 ARDS genes from genome and transcriptome studies that are involved in key inflammatory pathways [7], supporting a fundamental understanding of ARDS development. However, single nucleotide polymorphisms (SNPs) related to ARDS derived from genome-wide association studies (GWAS) were confined to European populations, except for one study we launched in African Americans [8]. This narrow ancestry sampling and conventional analysis limit the discovery of biological clues about ARDS pathophysiology.

ARDS has heterogeneous etiology but is characterized by acute widespread inflammation in the lung commonly precipitated by sepsis or pneumonia $[1,9]$. Similarly, severe acute respiratory syndrome coronavirus 2 (SARS-CoV-2) infection induces aggressive inflammatory and immune responses that damage the respiratory tract, often causing ARDS as COVID-19 progresses $[10,11]$. Recently, two COVID-19 GWAS preliminarily revealed host genetic components involved in the severe progression $[12,13]$, opening new avenues to elucidate COVID-19 pathophysiology, including ARDS development. Effective treatments for ARDS specifically caused by COVID-19, outside of supportive care interventions, remain elusive $[14,15]$. Therefore, meeting the urgent need for effective ARDS therapies necessitates the identification of new therapeutic targets.

To investigate the genetic architecture and molecular factors related to ARDS development, we applied integrative omics approaches and performed Mendelian Randomization analysis to infer candidate causal factors (Fig. 1). Our findings aid in the understanding of the progressive course of ARDS and identify potential targets for therapeutic development.

\section{Methods}

\section{Study participants}

Participants were recruited from the iSPAAR (Identification of SNPs Predisposing to Altered Acute Lung Injury Risk) consortium [16], MESSI (Molecular Epidemiology of Sepsis in the ICU) cohort $[17,18]$, and a study by Garcia et al. [8]. Details about population demographics and

\section{Take-home message}

Integration analyses of genome and transcriptome data reveal a novel functional locus possibly involved in the regulation of immune-inflammatory response in ARDS pathophysiology, and causal inference indicates several clinical interventions of ARDS development. Our findings inform further investigation of hostpathogen biology and therapeutic targets for ARDS, especially during the COVID-19 pandemic

data cleaning are described in Supplementary Methods, Table E1, and Figure E1-E3.

\section{Genetic association analysis and meta-analysis}

GWAS was conducted separately for each ARDS cohort across European and African American ancestries. Logistic regression was performed in an additive genetic model with adjustments for age, sex, and top three population ancestry principal components. Odds ratios (ORs) and corresponding 95\% confidence intervals (CIs) were calculated to estimate genetic effects. Association statistics from each ancestry group were combined separately using fixed meta-analysis with inverse-variance weighting of log-ORs via METAL [19], following the descriptor:./metal<script $>$, in which the commands of MARKER, ALLELE, WEIGHT, EFFECT, STDERR, FREQ, PROCESS, and PVAL were used to describe the input files across each study, and the commands of OUTFILE and ANALYSIS performed the final analysis.

\section{RNA sequencing (RNA-Seq) analysis}

Blood samples were collected from 160 ARDS cases and 142 controls (in-house dataset) for RNA-Seq analysis, of which 46 were matched to genotyping data for expression quantitative trait loci (QTL) analyses. Procedures of detection and quantitation are described in Supplementary Methods. Briefly, 19,898 protein-coding genes were identified for transcriptome analyses, including analyses of differential expression, pathway enrichment, and immune cell decomposition. Details for reanalysis of publicly supported COVID-19 single-cell RNA-Seq datasets are described in Supplementary Methods.

\section{Mendelian randomization (MR) analysis}

Two-sample MR analysis was conducted to obtain causal estimates of exposures on outcome using the TwoSampleMR R package [20]. Exposures empirically included 114 traits as key clinical issues of COVID19, grouped by disease, hematology, biochemistry, coagulation, nutrition or habit, and treatment (Table E2). Genetic instruments of each exposure were obtained from GWAS summary statistics in Europeans 

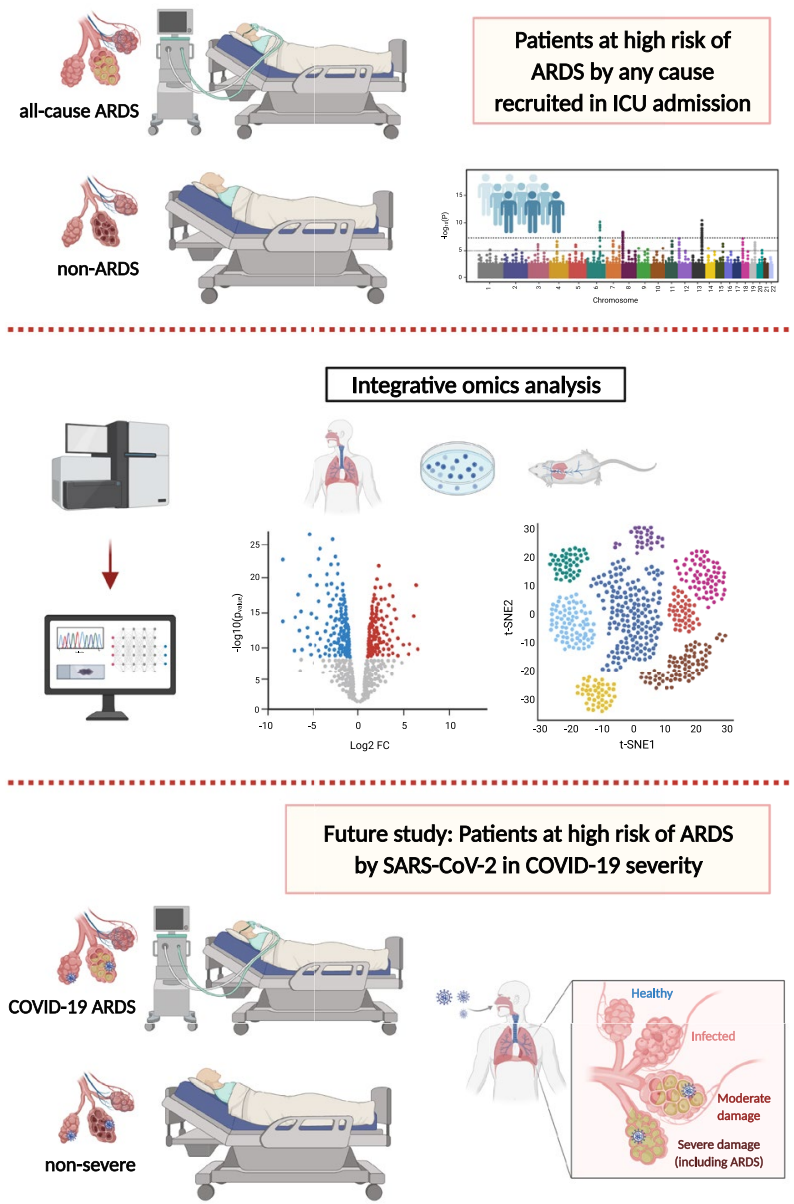

GWAS meta-analysis of ARDS across European and African American populations

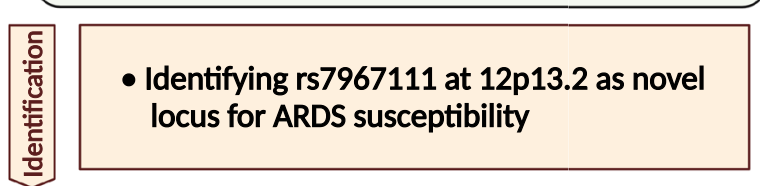

In silico analysis accompanied by transcriptome to decipher biologcial effects of rs7967111 and corresponding genes BORCS5 and DUSP16

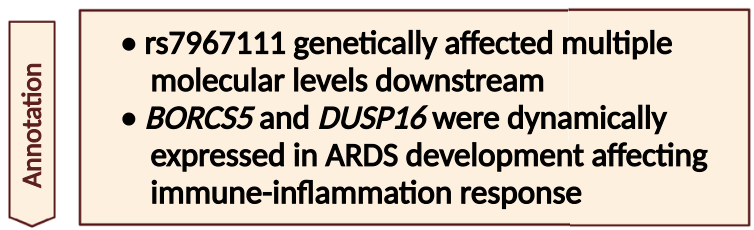

Transferability of all-cause ARDS findings in clinic

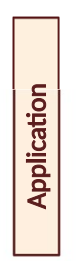

- BORCS5 and DUSP16 were decresased in immune cells alongside COVID-19 severity

- Putative causality in clinical observations may guide the ARDS care management

- Future investigation specific to COVID-19 ARDS is warranted

Fig. 1 Flowchart of study design. First, in the identification phase, ancestry-specific and trans-ancestry GWAS meta-analyses of ARDS were performed to compare two distinct ancestry populations to identify novel susceptibility genes for all-cause ARDS. Second, in the annotation phase, in silico analyses combined with transcriptome data were used to functionally annotate novel loci to decipher corresponding biological and genetic effects on ARDS pathophysiology. Last, in the application phase, a certain omics features and clinical observations were shared between all-cause ARDS and COVID-19 severity, suggesting clinical care of ARDS development, possibly as COVID-19 progresses. Flowchart was created online with BioRender.com

(Supplementary Methods). The outcome was ARDS using GWAS summary statistics in Europeans, generated by meta-analysis. Inverse-variance-weighted (IVW), weighted median, and MR-Egger regression were primarily used to calculate effect size $(\beta)$ and corresponding standard error (SE). The Wald ratio method was used if only one genetic instrument remained. Heterogeneity was estimated using MR-Egger and IVW methods. Directional pleiotropy was estimated via MREgger intercept test. Evidence score was calculated by aggregating the significance.

\section{Statistical analysis}

Analytical steps throughout the study are shown in Figure E2. In silico analyses are detailed in Supplementary Methods, including genetic heritability and correlation (via Genome-wide Complex Trait Analysis [GCTA], LD Score Regression [LDSC], and LD Hub), gene set analysis (via Multi-marker Analysis of GenoMic Annotation [MAGMA] and Data-driven Expression-Prioritized Integration for Complex Traits [DEPICT]), genetic function annotation (via Functional Annotation of VariantsOnline Resource [FAVOR]), phenome-wide association studies annotation (PheWAS; via SAIGE UKB and PhenomeXcan), expression pattern analyses (via Human Protein Atlas [HPA], Cancer Cell Line Encyclopedia 
[CCLE], Database of Immune Cell Expression, Expression quantitative trait loci and Epigenomics [DICE], UCSC Cell Browser, and Gene Expression Omnibus [GEO]), immune cell estimation (via CIBERSORTx), and polygenic risk score (PRS). Linear regression analysis, Kruskal-Wallis test, Wilcoxon signed-rank test, and Spearman rank correlation analysis were conducted as appropriate. All statistical analyses were done in R (version 3.5.1).

\section{Results}

\section{Genetic architecture of ARDS in European and African American populations}

We first conducted ancestry-specific GWAS metaanalyses for ARDS. In iSPAAR and MESSI European cohorts comprising 1250 cases and 1583 controls with 5,749,543 common variants (Fig. E2), rs7967111 A > G had a marginal effect of genome-wide significance on increasing risk of ARDS $(\mathrm{OR}=1.35,95 \% \mathrm{CI}=1.21-1.51$, $P=6.64 \times 10^{-8}$; Table E3 and Fig. E4A). For African American ancestry, we included 387 cases and 387 controls from MESSI and Garcia et al. cohorts with 6,255,902 variants (Fig. E2) and observed a top signal of rs619652 $\mathrm{A}>\mathrm{G}$ reaching a nominal significance $(\mathrm{OR}=1.86,95 \%$ $\mathrm{CI}=1.48-2.35, P=1.59 \times 10^{-7}$; Table E3 and Fig. E4A). Globally, the estimated genetic heritability of ARDS was higher in Europeans (GCTA: $h^{2}{ }_{\mathrm{SNP}}=0.129, \mathrm{SE}=0.023$; LDSC: $\left.h_{\text {SNP }}^{2}=0.126, \mathrm{SE}=0.049\right)$ than African Americans $\left(\mathrm{GCTA}: h_{\mathrm{SNP}}^{2}=0.039, \mathrm{SE}=0.032\right)$.

At the single-variant level, no significant loci were shared across ancestries (empirical threshold at $P<1 \times 10^{-4}$; Table E4 and Fig. E4B), even at the geneset level analyzed via MAGMA (Fig. E4C). Nevertheless, gene set enrichment analysis via DEPICT identified 45 ancestry-shared reconstituted enrichment sets (Fig. E4D), such as immune response activation (top in Europeans). The genetic correlation of ARDS between the two populations was 0.266 , but the large SE (0.589) caused by the small sample size limits interpretation. These findings suggest a distinct genetic heterogeneity of ARDS between ancestries, but some shared genetic components.

Thus, we performed a trans-ancestry GWAS metaanalysis. Unexpectedly, no loci achieved genomewide significance, even at the empirical threshold of $P<1 \times 10^{-6}$ (Fig. E5). Notably, rs7967111 from Europeans maintained a statistically significant association with ARDS $\left(\mathrm{OR}=1.26,95 \% \mathrm{CI}=1.14-1.39, P=4.38 \times 10^{-6}\right)$, while rs619652 from African Americans was not validated in trans-ancestry populations and had dramatic heterogeneity-even its minor allele was exchanged from $\mathrm{G}$ in Europeans to A in African Americans (Table E3).

To confirm the genetic association of rs7967111, we performed sensitivity analysis by requiring individual genotyping data from two European cohorts. Intriguingly, rs7967111 exceeded genome-wide significance $\left(P<5 \times 10^{-8}\right)$, with OR of $1.38\left(P=2.15 \times 10^{-8}\right.$; Table 1$)$ after adjusting for potential confounders. Thus, we retained rs7967111 for further analysis.

\section{Functional analysis of rs7967111 at $12 \mathrm{p} 13.2$}

Functional annotation showed that rs7967111 was located at the third intron of BORCS5 and approximately $20 \mathrm{~kb}$ downstream of DUSP16 within a high-LD region (Fig. E6A,B), harboring strong functional signals of enhancer activity, histone modification, and transcription factor binding calculated by FAVOR (Fig. E6C). Subsequently, genetic pleiotropy analysis indicated that rs7967111 may dysregulate BORCS5 and DUSP16 expression in blood (in-house dataset, Fig. 2a) and lung tissues (public datasets, Table E5) and alter neighboring methylation level at CpG site cg19207364, surrounding H3K27ac modification activity, and 15 blood metabolites abundance (Table E5). However, rs7967111 did not affect 11 ARDS protein biomarkers (Fig. E7). PheWAS for pleiotropy evaluation via SAIGE UKB and PhenomeXcan revealed that rs7967111 was dramatically associated with multiple phenotypes, especially in the respiratory

Table 1 Association between rs7967111 and ARDS in European populations

\begin{tabular}{|c|c|c|c|c|c|c|c|c|}
\hline Variant & Chr & BP & Locus & Non-effect/effect allele & EAF & $\begin{array}{l}\text { Multiple models for genetic } \\
\text { association }\end{array}$ & OR $(95 \% \mathrm{CI})$ & $P$ value \\
\hline \multirow[t]{5}{*}{ rs7967111 } & 12 & $12,601,953$ & BORCS5 intronic & $\mathrm{A} / \mathrm{G}$ & 0.444 & Model 1: rs7967111 genotypes & $1.30(1.17-1.45)$ & $9.67 \times 10^{-7}$ \\
\hline & & & & & & Model 2: model $1+$ age + sex & $1.33(1.19-1.48)$ & $2.57 \times 10^{-7}$ \\
\hline & & & & & & Model 3: model 2+ PCs & $1.35(1.21-1.50)$ & $7.63 \times 10^{-8}$ \\
\hline & & & & & & Model 4: model 3 + cohort & $1.36(1.22-1.52)$ & $3.58 \times 10^{-8}$ \\
\hline & & & & & & Model 5: model 4+ pneumonia & $1.38(1.23-1.55)$ & $2.15 \times 10^{-8}$ \\
\hline
\end{tabular}

ARDS acute respiratory distress syndrome; $C h r$ chromosome; $B P$ base pair position in $\mathrm{GRCh} 37 / \mathrm{hg} 19 ; \mathrm{OR}$ odds ratio; $\mathrm{Cl}$ confidence interval; $E A F$ effect allele frequency; $P C$ s population ancestry principal components $1-3$

$\mathrm{OR}, \mathrm{Cl}$, and $P$-values were calculated using logistic regression model with adjustments for confounders as appropriate. The result of model 3 was equivalent to $\mathrm{GWAS}$ meta-analysis in Europeans 


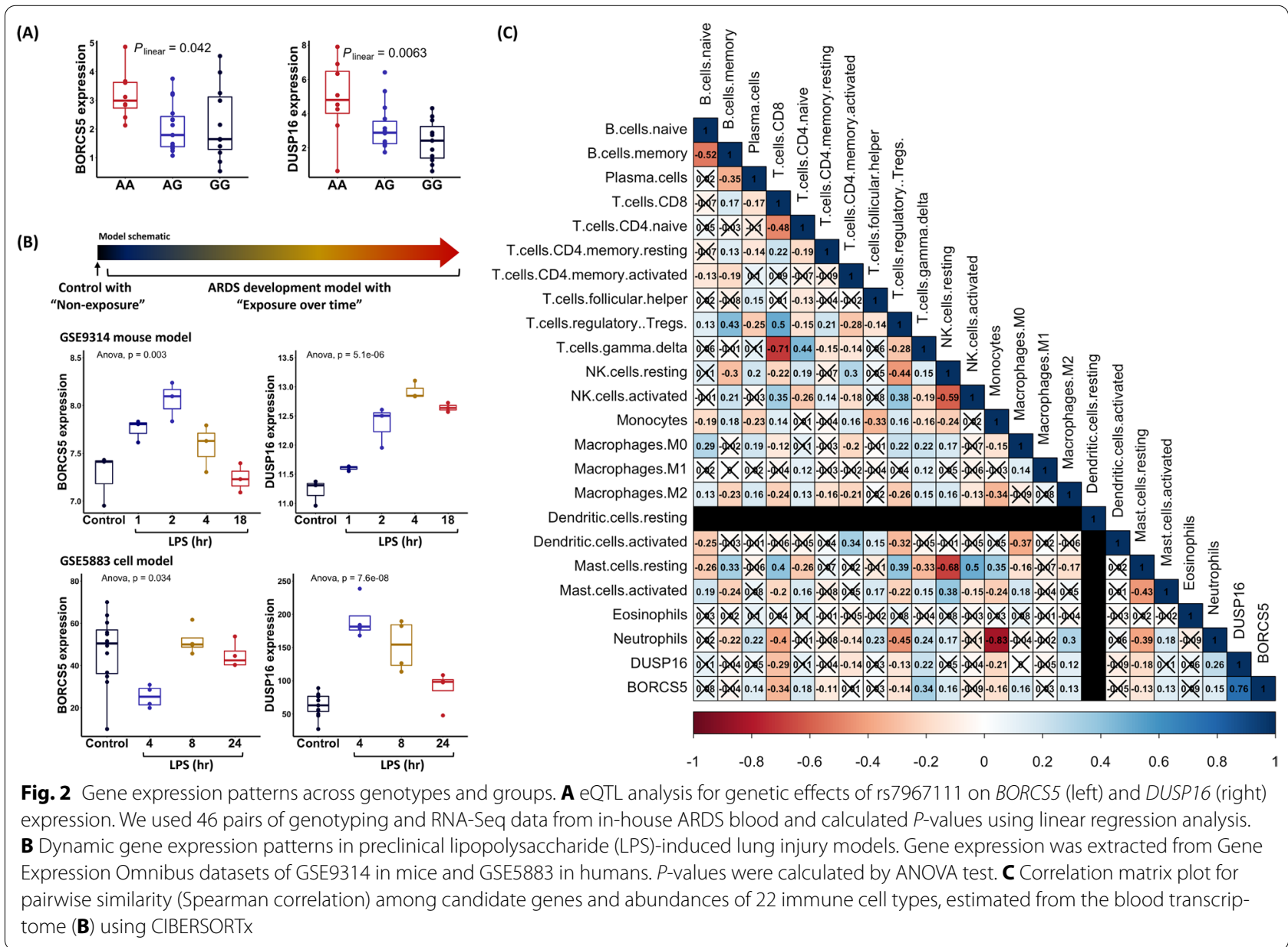

system (Fig. E8), and both BORCS5 and DUSP16 were significantly correlated with more than 100 phenotypes (Fig. E9). These results suggest that rs7967111 at 12p13.2 serves as a novel functional locus in ARDS development.

\section{Expression pattern and biological effect of susceptibility genes}

BORCS5 and DUSP16 were expressed across tissues at both protein and RNA levels (HPA database, Fig. E10). In the in-house dataset of 160 ARDS cases and 142 controls, BORCS5 and DUSP16 expression were highly correlated $(r=0.76, P<0.001$; Fig. E11A) regardless of case status (Fig. E11B). Strong correlations were also identified in cell lines, particularly in lung cells $\left(r_{\text {all }}=0.44\right.$, $P_{\text {all }}<0.001 ; r_{\text {lung }}=0.51, P_{\text {lung }}<0.001 ;$ CCLE dataset, Fig. E11C) and immune cells $(r>0.95, P<0.001$; DICE dataset, Fig. E11D).

Similar expression patterns were found at single-cell levels using the UCSC Cell Browser. BORCS5 (6.4\% of cells) and DUSP16 (14.5\%) were widely spread over nine cell types originating from lung tissues (Fig. E12A), especially enriched across primary bronchial epithelial cells (3.2\% and $1.1 \%$, respectively; Fig. E12B). Notably, BORCS5 and DUSP16 were highly expressed in pulmonary immune cells, including macrophages and dendritic cells (Fig. E12C).

Preclinical models of lung injury induced by lipopolysaccharide (LPS) retrieved from GEO were applied to assess the potential biological effects of candidate genes in ARDS pathobiology. Intriguingly, dynamic expression patterns of BORCS5 and DUSP16 were observed in both mouse lung tissues (GSE9314) and human lung microvascular endothelial cells (GSE5883) exposed to LPS over time (Fig. 2b). Both BORCS5 and DUSP16 showed greater expression in the first $4-8 \mathrm{~h}$ after LPS exposure, but then their expression decreased dramatically.

Immune characteristics of ARDS by transcriptome profiling We next profiled the blood transcriptome for 160 ARDS cases and 142 controls. There yielded 142 differentially expressed genes (112 upregulated and 30 
downregulated; Fig. E13A), and these represented enrichment for key immune pathways including Tolllike receptor signaling, CD8+ $\mathrm{T}$ cells, and immunodeficiency disease via gene set enrichment analysis (Fig. E13B-D). Further, among 22 immune cell types decomposed in the transcriptome via CIBERSORTx, 5 immune cell types were significantly increased in ARDS cases (Fig. E14). Remarkably, BORCS5 and DUSP16 were dramatically positively correlated with gamma delta $\mathrm{T}$ cells, M2 macrophages, and neutrophils, and negatively with $\mathrm{CD} 8+\mathrm{T}$ cells, regulatory $\mathrm{T}$ cells, monocytes, and resting mast cells (Fig. 2c). However, rs7967111 did not significantly influence these cell fractions (Fig. E15).

\section{Transferability of all-cause ARDS findings}

Given ARDS is a complication during the severe progression of COVID-19, we attempted to assess the transferability of all-cause ARDS findings (defined in this study) to understand COVID-19 severity. Genetically, three severe COVID-19 relevant SNPs (i.e., rs657152, rs10735079, and rs2109069) [12, 13] displayed consistent associations with all-cause ARDS development in this study, of which 2 were nominally significant $(P=0.040,0.013$, and 0.045 , respectively; Fig. E16). Intriguingly, the PRS calculated by COVID19 severity GWAS was significantly higher in ARDS cases than controls (Fig. 3a). In single-cell transcriptome profiles, BORCS5 and DUSP16 expression were significantly decreased or on a downward trend aligning with severe progression of COVID-19 in dendritic cells derived from upper respiratory tract samples (Fig. 3b) or peripheral blood mononuclear cells (Fig. 3c) $[21,22]$.

\section{Putative causal relationship between traits and ARDS}

Moreover, we estimated causal effects of 114 candidate risk factors derived from clinical observations of COVID-19 under the MR analytic framework (Fig. 4a). Six associations with evidence scores $\geq 2$ are shown in Fig. 4b. Notably, inflammatory bowel disease (IBD) and immune/inflammatory biomarkers [i.e., C-reactive protein (CRP), interleukin-10 (IL-10), and immunoglobulin
G index levels in cerebrospinal fluid] were causally associated with increased risk of ARDS development (all $\beta>0 ; \quad P_{\mathrm{IVW}}=0.027,0.015,0.002$, and $<0.001$, respectively), while daily supplements of vitamin D ( $\beta_{\text {MR- }}$ Egger $\left.=-25.80, P_{\text {MR-Egger }}=0.001\right)$ and use of vasodilators to treat cardiac diseases $\left(\beta_{\mathrm{IVW}}=-0.25, P_{\mathrm{IVW}}=0.031\right)$ were associated with decreased likelihood of severe progression (Table E2). Intriguingly, 47 of 855 traits were genetically correlated with ARDS in Europeans using LD Hub (Table E6), including IBD.

\section{Discussion}

To our knowledge, this is the first study to integrate transancestry GWAS in Europeans and African Americans, transcriptome analyses across bulk tissues and single cells, and preclinical models by mice and cells, to reveal a novel locus at 12p13.2 harboring rs7967111 as genetic predictor of ARDS susceptibility, BORCS5 and DUSP6 involved in heterogeneous etiologies of ARDS, and potential clinical interventions of ARDS development.

Initially, we launched a large-scale ARDS GWAS analysis comprising European and African American populations. Although a distinct genetic architecture of ARDS exists across ancestries, gene set-based inference eliminated ancestry specificity, which showed 45 shared functional elements in ARDS etiology, including immune response. Trans-ancestry GWAS meta-analysis improves the resolution of genetic effects on phenotypes [23]. However, we did not observe signals passing genomewide significance when combining the two ancestries, possibly because of the limited sample size and varied ethnic background of African Americans. Thus, a largescale non-European population for ARDS genomic studies was advocated to further maximize genetic discovery and reduce health disparities.

Nevertheless, rs7967111 at 12p13.2 presented a significant genome-wide effect on increasing ARDS risk after sensitivity analyses. The subsequent pleiotropic evaluation indicated that rs7967111 was a potential causal variant of ARDS by disturbing a cascade of DNA methylation, histone modification, gene expression, and metabolites. PheWAS is well suited to facilitate pleiotropic evaluation of novel risk SNPs on phenotypes [24]. Our

(See figure on next page.)

Fig. 3 Omics transferability assessment of all-cause ARDS. A Differential polygenic risk score (PRS) between ARDS cases and controls. PRS was calculated via weighting effect size derived from three severe COVID-19 GWAS assigned to each ARDS case and control in Europeans. $P$-value was calculated via t-test. B,C Differential expression analysis for BORCS5 and DUSP16 in dendritic cells derived from two single-cell RNA-Seq datasets of upper respiratory tract samples [21] (B) and peripheral blood mononuclear cells [22] (C) in COVID-19 patients and healthy controls, respectively. Dots represent cells expressing candidate genes and are colored by the severity of COVID-19. Healthy samples from (B) dataset were removed because only one cell expressed candidate genes. P-values were calculated from a Wilcoxon test. The $y$-axis is on a log-10 scale to show gene expression, and " $n$ " indicates the number of cells detected with candidate gene expression. The solid line of each plot indicates the median of gene expression for each group, and box edges mark lower and upper quartiles of gene expression 
(A)

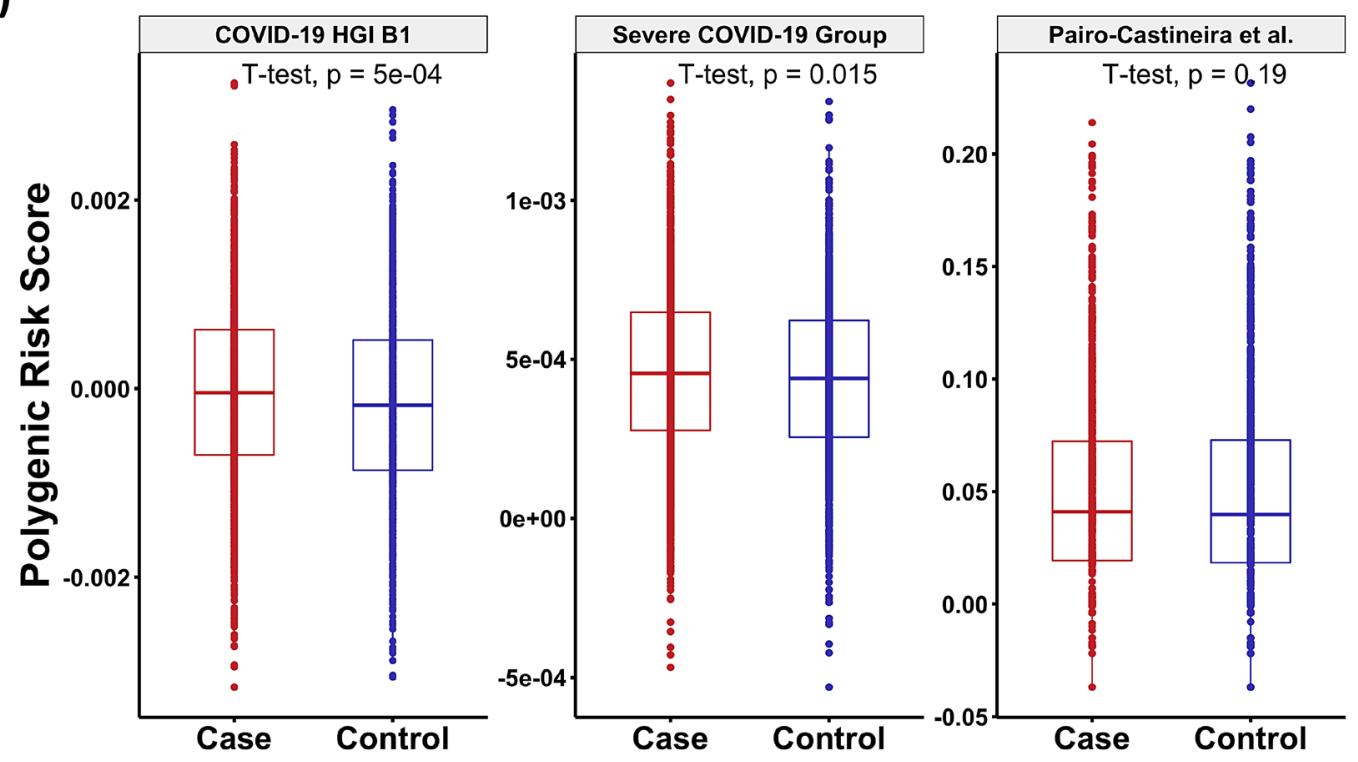

(B)

Dendritic Cells in Upper Respiratory Tracts
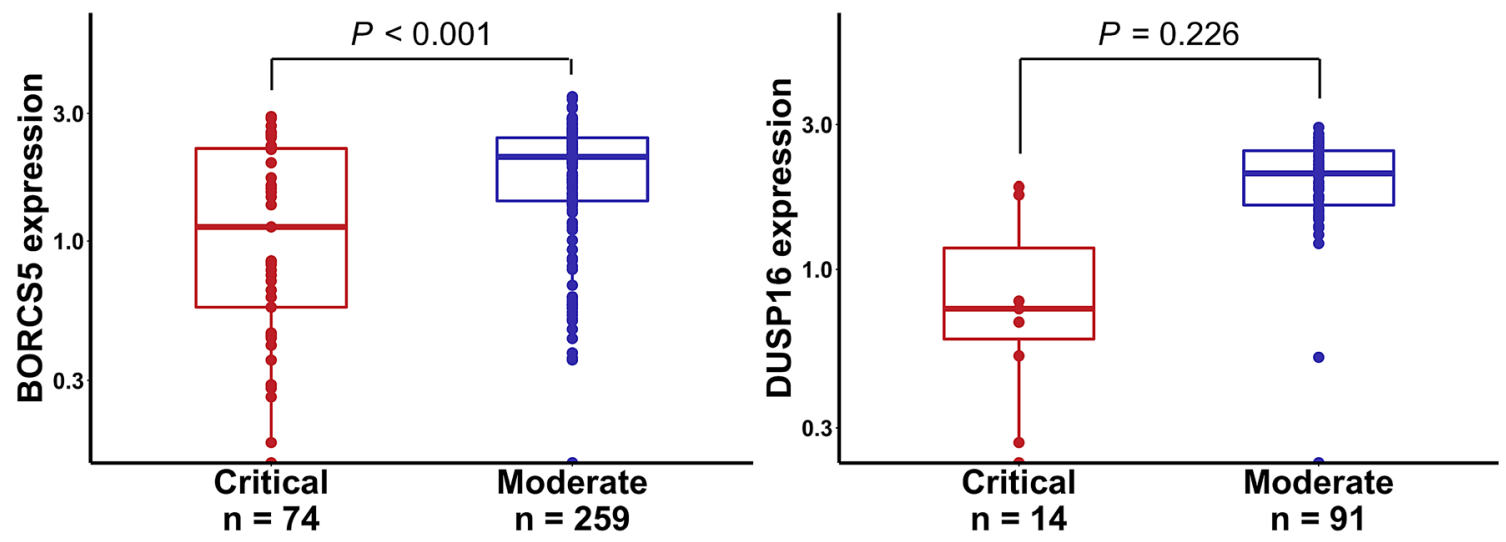

(C)

Dendritic Cells in Peripheral Blood Mononuclear Cells
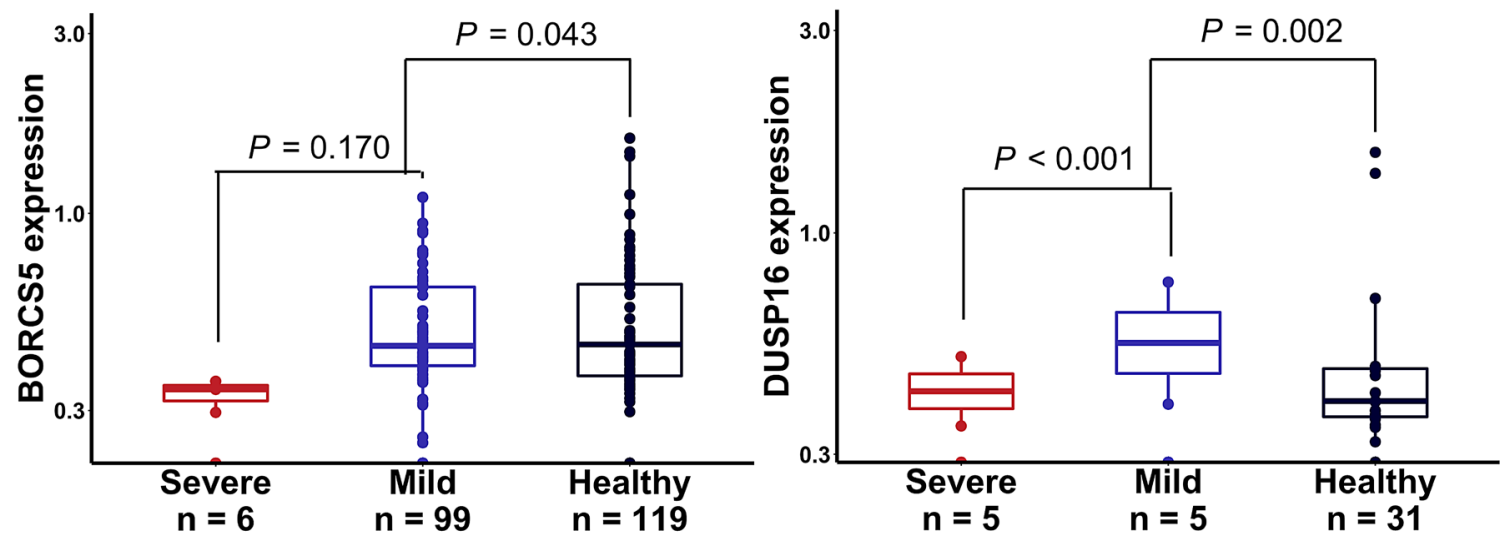
(A)

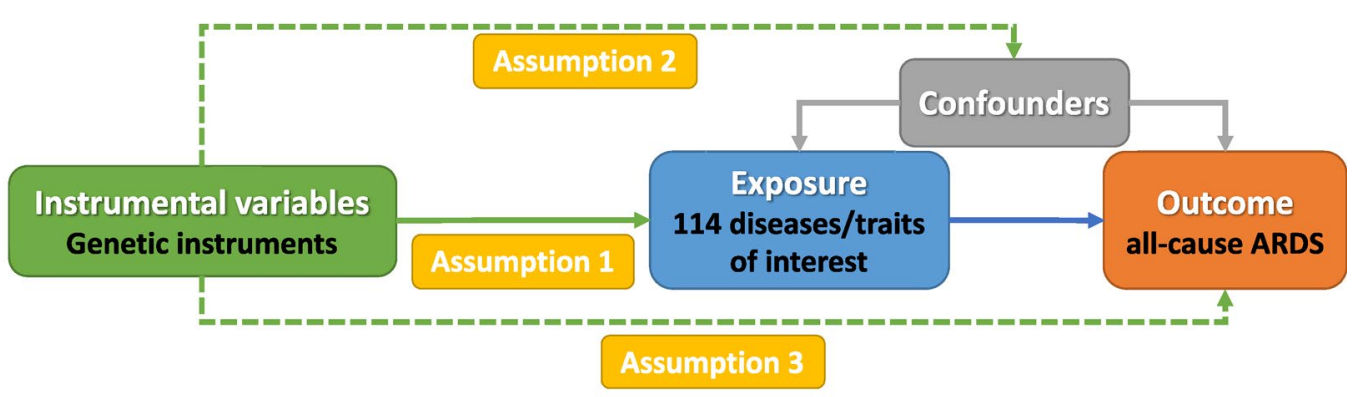

(B)
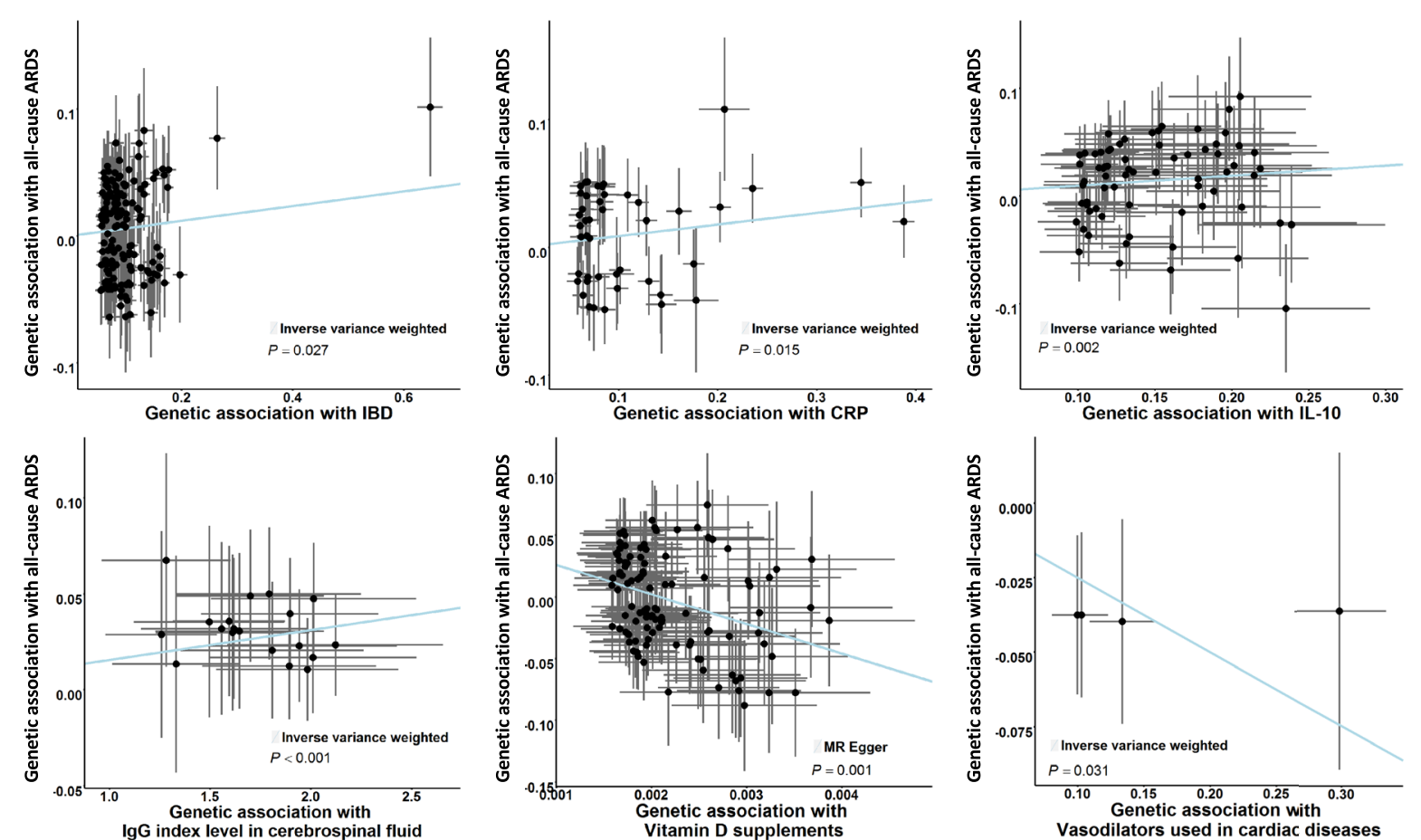

Fig. 4 Results of Mendelian Randomization analysis for all-cause ARDS. a Schematic diagram for assumptions of Mendelian Randomization analysis. Assumption 1: genetic instruments selected should be robustly associated with the exposure, usually underlying $P<5 \times 10^{-8}\left(P<1 \times 10^{-6}\right.$ or $P<1 \times 10^{-4}$ as suggestive significance level); Assumption 2: genetic variants should not be associated with potential confounders; and Assumption 3: genetic variants of an exposure should affect the outcome risk only through the risk factor, not via alternative pathways. b Scatter plots for putative causal associations between six diseases/traits and all-cause ARDS under evidence score of $\geq 2$. P-values were calculated with IVW or MR-Egger regression

analyses further indicated that rs7967111 and the annotated genes BORCS5 and DUSP16 displayed biological roles in multiple traits or diseases, especially those referring to the lung.

The following transcriptome analyses spanning bulk tissues and single cells dissected their functions in ARDS that BORCS5 and DUSP16 were not only highly correlated mutually in pulmonary immune cells but also influenced cell fractions of immune CD8+ T cells, regulatory $\mathrm{T}$ cells, and macrophages in blood, separately from genetic effects of rs7967111. Notably, preclinical models analyses revealed that BORCS5 and
DUSP16 expression exhibit dynamic change but ultimately decrease during ARDS development. Physiologically, BORCS5 recruits ARL8B to promote lysosome movement and reduce cell spreading and migration [25, 26]; and DUSP16 inactivates MAP kinases to regulate cellular senescence important for immune responses $[27,28]$. These comprehensive analyses indicate that rs7967111 was a functional genetic predictor for ARDS susceptibility via disrupting both BORCS5 and DUSP16 expression, among which the latter served as regulatory factors affecting immune response in ARDS pathophysiology under certain causes. 
Emerging studies propose that it is reasonable to borrow concepts from other-cause ARDS pathogenesis to inform evolving understanding of COVID-19 ARDS in severe progression while awaiting more diseasefocused data [29]. Clinically, patients who progress to severe COVID-19 meet ARDS diagnosis criteria [30], and represent a specific subset of all-cause ARDS with clinical similarity in respiratory mechanics, therapeutic response, and prognosis [31, 32]. Therefore, we hypothesized whether the available data on all-cause ARDS could glean insights into ARDS development as COVID19 progresses. In this study, we observed that several genetic components in COVID-19 severity $[12,13]$ were shared in all-cause ARDS and the corresponding PRS could distinguish ARDS cases from controls. Biologically, an intriguing observation from single-cell transcriptome data showed gradually reduced expression of both BORCS5 and DUSP16 in dendritic cells correlated with COVID-19 severity, which was in accordance with the expression pattern in preclinical models of ARDS development. Together, these features may suggest mechanisms that BORCS5 and DUSP16 may be involved in COVID-19 severity as similar to the immune-inflammation response in ARDS pathophysiology.

Furthermore, we observed some clinically instructive results by MR analysis. Elevated levels of CRP and IL-10 were commonly happened in severe COVID-19 patients [33], and IBD patients were at higher risk of developing severity [34]; in contrast, vasodilator use and vitamin D supplements might reduce the risk of COVID-19 infections and mortality $[35,36]$. These clinical observations were consistent with their causal associations with ARDS development, which may guide the ARDS care management, especially during COVID-19 severe progression.

We acknowledge several limitations of this study. First, the transcriptome for all-cause ARDS was derived from blood, possibly explaining non-significant differential expression of BORCS5 and DUSP16. Further studies applying lung-specific transcriptomes of ARDS (including COVID-19 ARDS) will inform future treatment strategies. Second, it is necessary to perform large-scale ARDS GWAS in diverse populations to confirm our findings, especially for the transferability of all-cause ARDS to COVID-19 ARDS in severe progression. The generalizability of these results warrants further omics validation by recruiting larger populations of patients with COVID-19 with and without ARDS. Third, MR associations are subject to pleiotropic effects of genetic instruments, such as treatments in IBD and all-cause ARDS, which might be associated with some unexpected hidden confounders in GWAS summary statistics calculations. Therefore, well-powered randomized trials are needed to conclusively evaluate this association.
In summary, we uncovered a novel locus at $12 \mathrm{p} 13.2$ encompassing rs7967111 and functional involvement of BORCS5 and DUSP16 in ARDS development via immune-inflammatory processes. A certain extent of shared molecular characteristics and clinical observations indicate the leverage of findings in predicting ARDS susceptibility, elucidating ARDS pathophysiology, and informing treatment and intervention strategies for ARDS development, including those induced by COVID-19.

\section{Supplementary Information \\ The online version contains supplementary material available at https://doi. org/10.1007/s00134-021-06410-5.}

\section{Author details}

${ }^{1}$ Department of Environmental Health, Harvard T.H. Chan School of Public Health, Harvard University, 655 Huntington Avenue, Boston, MA 02115, USA. 2 Department of Biostatistics, Center for Global Health, School of Public Health, Nanjing Medical University, 101 Longmian Avenue, Nanjing 211166, Jiangsu, China. ${ }^{3}$ Department of Medicine, University of Arizona, Tucson, AZ, USA. ${ }^{4}$ Pulmonary, Allergy, and Critical Care Medicine Division, University of Pennsylvania Perelman School of Medicine, Philadelphia, PA, USA. ${ }^{5}$ Department of Genetic Toxicology, The Key Laboratory of Modern Toxicology of Ministry of Education, Center for Global Health, School of Public Health, Nanjing Medical University, 101 Longmian Avenue, Nanjing 211166, Jiangsu, China. ${ }^{6}$ Department of Environmental Health Sciences, Arnold School of Public Health, University of South Carolina, Columbia, SC, USA. ${ }^{7}$ China International Cooperation Center for Environment and Human Health, Nanjing Medical University, Nanjing, Jiangsu,

China. ${ }^{8}$ Department of Biostatistics, Harvard T.H. Chan School of Public Health, Harvard University, Boston, MA, USA. ${ }^{9}$ Department of Statistics, Harvard University, Cambridge, MA, USA. ${ }^{10}$ The Affiliated Suzhou Hospital of Nanjing Medical University, Suzhou Municipal Hospital, Gusu School, Nanjing Medical University, Nanjing, Jiangsu, China. ${ }^{11}$ Pulmonary and Critical Care Unit, Department of Medicine, Massachusetts General Hospital/Harvard Medical School, Boston, MA, USA.

\section{Acknowledgements}

The authors acknowledge the patients of the ARDS cohorts who graciously agreed to participate in this research study. They also acknowledge the nurses, physicians, and staff in the medical and surgical ICUs who participated in the clinical care of the enrolled patients. They also thank Dr. Mark M. Wurfel (University of Washington, Seattle, WA, USA) for his efforts in co-leading the iSPAAR cohort.

\section{Author contributions}

M.L.D. and D.C.C. conceived the study design and had full access to the data. M.L.D. took responsibility for the integrity of data and accuracy of the analyses. D.C.C., J.G.G., J.D.C., Z.Z.Z., P.T., N.J.M., and M.L.W. organized and entered data. M.L.D., J.Y.X., G.S.C., S.P.S., X.S.D., H.L., and J.N.H. contributed to data analyses. M.L.D., J.Y.X., Q.Y.Y., Z.D.Z., F.C., M.L.W., and X.H.L. contributed to data interpretation. M.L.D. and D.C.C. drafted the manuscript. All authors made significant contributions to the final manuscript and approve its submission.

\section{Funding}

This study was supported by the US National Institutes of Health (R01HL060710, R56HL134356, and ES000002 to D.C.C.) and the Special Program of the National Natural Science Foundation of China (NSFC) on tracing, pathogenesis, prevention, and treatment for COVID-19 (82041024 to F.C.).

\section{Availability of data and materials}

For the iSPAAR consortium dataset, the genotype data and relevant covariate information (age, sex, ancestry, principal components, etc.) are deposited in dbGaP under accession codes phs000631.v1.p1. For MESSI and the African American dataset, dbGaP submission is forthcoming in accordance with the 
$\mathrm{NIH}$ genomic data sharing policy. In advance of their availability on dbGaP, full summary statistics are available on request to the authors.

\section{Code availability}

Not applicable.

Declarations

\section{Conflicts of interest}

The authors state that there is no conflict of interest.

\section{Ethical approval}

The study protocol of each cohort was approved by the institutional review board at each participating site.

\section{Consent to participate}

All participants provided written consent.

\section{Consent for publication}

All authors approve this study submission.

\section{Publisher's Note}

Springer Nature remains neutral with regard to jurisdictional claims in published maps and institutional affiliations.

Received: 26 September 2020 Accepted: 9 April 2021

Published online: 25 May 2021

\section{References}

1. Fan E, Brodie D, Slutsky AS (2018) Acute respiratory distress syndrome: advances in diagnosis and treatment. JAMA 319(7):698-710. https://doi. org/10.1001/jama.2017.21907

2. Bellani G, Laffey JG, Pham T, Fan E, Brochard L, Esteban A, Gattinoni L, van Haren F, Larsson A, McAuley DF, Ranieri M, Rubenfeld G, Thompson BT, Wrigge H, Slutsky AS, Pesenti A (2016) Epidemiology, patterns of care, and mortality for patients with acute respiratory distress syndrome in intensive care units in 50 countries. JAMA 315(8):788-800. https://doi. org/10.1001/jama.2016.0291

3. Network C-IGobotR, the C-ICUI (2020) Clinical characteristics and day-90 outcomes of 4244 critically ill adults with COVID-19: a prospective cohort study. Intensive Care Med. https://doi.org/10.1007/s00134-020-06294-x

4. Wang D, Hu B, Hu C, Zhu F, Liu X, Zhang J, Wang B, Xiang H, Cheng Z, Xiong Y, Zhao Y, Li Y, Wang X, Peng Z (2020) Clinical characteristics of 138 hospitalized patients with 2019 novel coronavirus-infected pneumonia in Wuhan, China. Jama. https://doi.org/10.1001/jama.2020.1585

5. Li X, Ma X (2020) Acute respiratory failure in COVID-19: is it "typical" ARDS? Crit Care 24(1):198. https://doi.org/10.1186/s13054-020-02911-9

6. Karczewski KJ, Snyder MP (2018) Integrative omics for health and disease. Nat Rev Genet 19(5):299-310. https://doi.org/10.1038/nrg.2018.4

7. Lynn H, Sun X, Casanova N, Gonzales-Garay M, Bime C, Garcia JGN (2019) Genomic and genetic approaches to deciphering acute respiratory distress syndrome risk and mortality. Antioxid Redox Signal 31(14):10271052. https://doi.org/10.1089/ars.2018.7701

8. Bime C, Pouladi N, Sammani S, Batai K, Casanova N, Zhou T, Kempf CL, Sun X, Camp SM, Wang T, Kittles RA, Lussier YA, Jones TK, Reilly JP, Meyer NJ, Christie JD, Karnes JH, Gonzalez-Garay M, Christiani DC, Yates CR, Wurfel MM, Meduri GU, Garcia JGN (2018) Genome-wide association study in African Americans with acute respiratory distress syndrome identifies the selectin P ligand gene as a risk factor. Am J Respir Crit Care Med 197(11):1421-1432. https://doi.org/10.1164/rccm.201705-09610C

9. Matthay MA, Arabi YM, Siegel ER, Ware LB, Bos LDJ, Sinha P, Beitler JR, Wick KD, Curley MAQ, Constantin JM, Levitt JE, Calfee CS (2020) Phenotypes and personalized medicine in the acute respiratory distress syndrome. Intensive Care Med. https://doi.org/10.1007/s00134-020-06296-9

10. Zou L, Ruan F, Huang $M$, Liang L, Huang H, Hong Z, Yu J, Kang M, Song $Y$, Xia J, Guo Q, Song T, He J, Yen HL, Peiris M, Wu J (2020) SARS-CoV-2 viral load in upper respiratory specimens of infected patients. N Engl J Med 382(12):1177-1179. https://doi.org/10.1056/NEJMc2001737

11. Cevik M, Kuppalli K, Kindrachuk J, Peiris M (2020) Virology, transmission, and pathogenesis of SARS-CoV-2. BMJ 371:m3862. https://doi.org/10. 1136/bmj.m3862

12. Ellinghaus $D$, Degenhardt $F$, Bujanda $L$, Buti $M$, Albillos $A$, Invernizzi $P$, Fernandez J, Prati D, Baselli G, Asselta R, Grimsrud MM, Milani C, Aziz F, Kassens J, May S, Wendorff M, Wienbrandt L, Uellendahl-Werth F, Zheng T, Yi X, de Pablo R, Chercoles AG, Palom A, Garcia-Fernandez AE, Rodriguez-Frias F, Zanella A, Bandera A, Protti A, Aghemo A, Lleo A, Biondi A, Caballero-Garralda A, Gori A, Tanck A, Carreras Nolla A, Latiano A, Fracanzani AL, Peschuck A, Julia A, Pesenti A, Voza A, Jimenez D, Mateos B, Nafria Jimenez B, Quereda C, Paccapelo C, Gassner C, Angelini C, Cea C, Solier A, Pestana D, Muniz-Diaz E, Sandoval E, Paraboschi EM, Navas E, Garcia Sanchez F, Ceriotti F, Martinelli-Boneschi F, Peyvandi F, Blasi F, Tellez L, Blanco-Grau A, Hemmrich-Stanisak G, Grasselli G, Costantino G, Cardamone G, Foti G, Aneli S, Kurihara H, ElAbd H, My I, GalvanFemenia I, Martin J, Erdmann J, Ferrusquia-Acosta J, Garcia-Etxebarria K, Izquierdo-Sanchez L, Bettini LR, Sumoy L, Terranova L, Moreira L, Santoro L, Scudeller L, Mesonero F, Roade L, Ruhlemann MC, Schaefer M, Carrabba M, Riveiro-Barciela M, Figuera Basso ME, Valsecchi MG, Hernandez-Tejero M, Acosta-Herrera M, D’Angio M, Baldini M, Cazzaniga M, Schulzky M, Cecconi M, Wittig M, Ciccarelli M, Rodriguez-Gandia M, Bocciolone M, Miozzo M, Montano N, Braun N, Sacchi N, Martinez N, Ozer O, Palmieri O, Faverio P, Preatoni P, Bonfanti P, Omodei P, Tentorio P, Castro P, Rodrigues PM, Blandino Ortiz A, de Cid R, Ferrer R, Gualtierotti R, Nieto R, Goerg S, Badalamenti S, Marsal S, Matullo G, Pelusi S, Juzenas S, Aliberti S, Monzani V, Moreno V, Wesse T, Lenz TL, Pumarola T, Rimoldi V, Bosari S, Albrecht W, Peter W, Romero-Gomez M, D'Amato M, Duga S, Banales JM, Hov JR, Folseraas T, Valenti L, Franke A, Karlsen TH, GG Severe Covid (2020) Genomewide association study of severe Covid-19 with respiratory failure. N Engl J Med. https://doi.org/10.1056/NEJMoa2020283

13. Pairo-Castineira E, Clohisey S, Klaric L, Bretherick AD, Rawlik K, Pasko D, Walker S, Parkinson N, Fourman MH, Russell CD, Furniss J, Richmond A, Gountouna E, Wrobel N, Harrison D, Wang B, Wu Y, Meynert A, Griffiths F, Oosthuyzen W, Kousathanas A, Moutsianas L, Yang Z, Zhai R, Zheng C, Grimes G, Beale R, Millar J, Shih B, Keating S, Zechner M, Haley C, Porteous DJ, Hayward C, Yang J, Knight J, Summers C, Shankar-Hari M, Klenerman P, Turtle L, Ho A, Moore SC, Hinds C, Horby P, Nichol A, Maslove D, Ling L, McAuley D, Montgomery H, Walsh T, Pereira A, Renieri A, Gen Ol, Investigators I, Initiative C-HG, Me I, Investigators B, Gen Cl, Shen X, Ponting CP, Fawkes A, Tenesa A, Caulfield M, Scott R, Rowan K, Murphy L, Openshaw PJM, Semple MG, Law A, Vitart V, Wilson JF, Baillie JK (2020) Genetic mechanisms of critical illness in Covid-19. Nature. https://doi.org/ 10.1038/s41586-020-03065-y

14. Ramanathan $K$, Antognini D, Combes A, Paden M, Zakhary B, Ogino M, MacLaren G, Brodie D, Shekar K (2020) Planning and provision of ECMO services for severe ARDS during the COVID-19 pandemic and other outbreaks of emerging infectious diseases. Lancet Respir Med 8(5):518-526. https://doi.org/10.1016/S2213-2600(20)30121-1

15. Goligher EC, Ranieri VM, Slutsky AS (2021) Is severe COVID-19 pneumonia a typical or atypical form of ARDS? And does it matter? Intensive Care Med 47(1):83-85. https://doi.org/10.1007/s00134-020-06320-y

16. National Heart $L, B l o o d$ Institute Acute Respiratory Distress Syndrome Clinical Trials N, Rice TW, Wheeler AP, Thompson BT, Steingrub J, Hite RD, Moss M, Morris A, Dong N, Rock P (2012) Initial trophic vs full enteral feeding in patients with acute lung injury: the EDEN randomized trial. Jama 307(8):795-803. https://doi.org/10.1001/jama.2012.137

17. Reilly JP, Wang F, Jones TK, Palakshappa JA, Anderson BJ, Shashaty MGS, Dunn TG, Johansson ED, Riley TR, Lim B, Abbott J, Ittner CAG, Cantu E, Lin X, Mikacenic C, Wurfel MM, Christiani DC, Calfee CS, Matthay MA, Christie JD, Feng R, Meyer NJ (2018) Plasma angiopoietin-2 as a potential causal marker in sepsis-associated ARDS development: evidence from Mendelian randomization and mediation analysis. Intensive Care Med 44(11):1849-1858. https://doi.org/10.1007/s00134-018-5328-0

18. Su L, Zhai R, Sheu CC, Gallagher DC, Gong MN, Tejera P, Thompson BT, Christiani DC (2009) Genetic variants in the angiopoietin-2 gene are associated with increased risk of ARDS. Intensive Care Med 35(6):1024-1030. https://doi.org/10.1007/s00134-009-1413-8 
19. Willer CJ, Li Y, Abecasis GR (2010) METAL: fast and efficient meta-analysis of genomewide association scans. Bioinformatics 26(17):2190-2191. https://doi.org/10.1093/bioinformatics/btq340

20. Hemani G, Zheng J, Elsworth B, Wade KH, Haberland V, Baird D, Laurin C, Burgess S, Bowden J, Langdon R, Tan VY, Yarmolinsky J, Shihab HA, Timpson NJ, Evans DM, Relton C, Martin RM, Davey Smith G, Gaunt TR, Haycock PC (2018) The MR-Base platform supports systematic causal inference across the human phenome. eLife 7. https://doi.org/10.7554/ elife.34408

21. Chua RL, Lukassen S, Trump S, Hennig BP, Wendisch D, Pott F, Debnath O, Thurmann L, Kurth F, Volker MT, Kazmierski J, Timmermann B, Twardziok S, Schneider S, Machleidt F, Muller-Redetzky H, Maier M, Krannich A, Schmidt S, Balzer F, Liebig J, Loske J, Suttorp N, Eils J, Ishaque N, Liebert UG, von Kalle C, Hocke A, Witzenrath M, Goffinet C, Drosten C, Laudi S, Lehmann I, Conrad C, Sander LE, Eils R (2020) COVID-19 severity correlates with airway epithelium-immune cell interactions identified by singlecell analysis. Nat Biotechnol 38(8):970-979. https://doi.org/10.1038/ s41587-020-0602-4

22. Lee JS, Park S, Jeong HW, Ahn JY, Choi SJ, Lee H, Choi B, Nam SK, Sa M, Kwon JS, Jeong SJ, Lee HK, Park SH, Park SH, Choi JY, Kim SH, Jung I, Shin EC (2020) Immunophenotyping of COVID-19 and influenza highlights the role of type I interferons in development of severe COVID-19. Sci Immunol 5 (49). https://doi.org/10.1126/sciimmunol.abd1554

23. Evangelou E, loannidis JP (2013) Meta-analysis methods for genomewide association studies and beyond. Nat Rev Genet 14(6):379-389. https://doi.org/10.1038/nrg3472

24. Hall MA, Verma A, Brown-Gentry KD, Goodloe R, Boston J, Wilson S, McClellan B, Sutcliffe C, Dilks HH, Gillani NB, Jin H, Mayo P, Allen M, Schnetz-Boutaud N, Crawford DC, Ritchie MD, Pendergrass SA (2014) Detection of pleiotropy through a phenome-wide association study (PheWAS) of epidemiologic data as part of the Environmental Architecture for Genes Linked to Environment (EAGLE) study. PLoS Genet 10(12):e1004678. https://doi.org/10.1371/journal.pgen.1004678

25. Pu J, Schindler C, Jia R, Jarnik M, Backlund P, Bonifacino JS (2015) BORC, a multisubunit complex that regulates lysosome positioning. Dev Cell 33(2):176-188. https://doi.org/10.1016/j.devcel.2015.02.011

26. Niwa S, Tao L, Lu SY, Liew GM, Feng W, Nachury MV, Shen K (2017) BORC regulates the axonal transport of synaptic vesicle precursors by activating ARL-8. Curr Biol 27(17):2569-2578 e2564. https://doi.org/10.1016/j.cub. 2017.07.013

27. Zhang H, Zheng H, Mu W, He Z, Yang B, Ji Y, Hui L (2015) DUSP16 ablation arrests the cell cycle and induces cellular senescence. FEBS J 282(23):4580-4594. https://doi.org/10.1111/febs.13518
28. Low HB, Zhang Y (2016) Regulatory roles of MAPK phosphatases in cancer. Immune Netw 16(2):85-98. https://doi.org/10.4110/in.2016.16.2.85

29. Torres Acosta MA, Singer BD (2020) Pathogenesis of COVID-19-induced ARDS: implications for an ageing population. Eur Respir J 56(3). https:// doi.org/10.1183/13993003.02049-2020

30. Berlin DA, Gulick RM, Martinez FJ (2020) Severe Covid-19. N Engl J Med. https://doi.org/10.1056/NEJMcp2009575

31. Chiumello D, Busana M, Coppola S, Romitti F, Formenti P, Bonifazi M, Pozzi T, Palumbo MM, Cressoni M, Herrmann P, Meissner K, Quintel M, Camporota L, Marini JJ, Gattinoni L (2020) Physiological and quantitative CTscan characterization of COVID-19 and typical ARDS: a matched cohort study. Intensive Care Med. https://doi.org/10.1007/s00134-020-06281-2

32. Brault C, Zerbib Y, Kontar L, Fouquet U, Carpentier M, Metzelard M, Soupison T, De Cagny B, Maizel J, Slama M (2020) COVID-19- versus nonCOVID-19-related acute respiratory distress syndrome: differences and similarities. Am J Respir Crit Care Med 202(9):1301-1304. https://doi.org/ 10.1164/rccm.202005-2025LE

33. Tay MZ, Poh CM, Renia L, MacAry PA, Ng LFP (2020) The trinity of COVID19: immunity, inflammation and intervention. Nat Rev Immunol. https:// doi.org/10.1038/s41577-020-0311-8

34. Derikx L, Lantinga MA, de Jong DJ, van Dop WA, Creemers RH, Romkens TEH, Jansen JM, Mahmmod N, West RL, Tan A, Bodelier AGL, Gorter MHP, Boekema PJ, Halet ERC, Horjus CS, van Dijk MA, Hirdes MMC, Epping Stippel LSM, Jharap B, Lutgens M, Russel MG, Gilissen LPL, Nauta S, van Bodegraven AA, Hoentjen F (2020) Clinical outcomes of COVID-19 in patients with inflammatory bowel disease: a nationwide cohort study. J Crohns Colitis. https://doi.org/10.1093/ecco-jcc/jjaa215

35. Shoaib MH, Ahmed FR, Sikandar M, Yousuf Rl, Saleem MT (2021) A journey from SARS-CoV-2 to COVID-19 and beyond: a comprehensive insight of epidemiology, diagnosis, pathogenesis, and overview of the progress into its therapeutic management. Front Pharmacol 12:576448. https:// doi.org/10.3389/fphar.2021.576448

36. Hu J, Zhang L, Lin W, Tang W, Chan FKL, Ng SC (2021) Review article: probiotics, prebiotics and dietary approaches during COVID-19 pandemic. Trends Food Sci Technol 108:187-196. https://doi.org/10.1016/j.tifs.2020. 12.009 